\title{
CONTAMINACIÓN AMBIENTAL E INFECCIONES RESPIRATORIAS EN NINOS
}

\author{
ENVIRONMENTAL POLLUTION AND RESPIRATORY INFECTIONS IN CHILDREN
}

Dra. Soledad Montes Franceschini

Broncopulmonar infantil Hospital Dra Eloisa Diaz - La Florida y Hospital Clínico San Borja Arriarán.

\section{INTRODUCCIÓN}

La Organización Panamericana de Salud estima que alrededor de 100 mil niños menores de 5 años en las Américas fallecen anualmente debido a peligros químicos, físicos y biológicos ambientales (1). Una de las primeras descripciones de la correlación entre la contaminación ambiental y morbi-mortalidad respiratoria fue en relación a la nube tóxica de humo de Londres en 1952. Sin embargo, recién la última década se ha evidenciado un aumento significativo de las publicaciones de la contaminación y los efectos deletéreos en la salud de las personas (2)

El impacto de la contaminación ambiental en la salud es muy variable y dependen del tipo de contaminante, pudiendo ser químicos, biológicos y físicos, además de factores propios del individuo, siendo más susceptibles los niños, mujeres embarazadas y personas de tercera edad.

El objetivo de este artículo, es evaluar como la contaminación ambiental aérea intra y extradomiciliaria se relaciona con las infecciones respiratorias en los niños. Es fundamental, considerar que gran parte de las actividades de la vida diaria en los países occidentales ocurren dentro del hogar, colegios y oficinas (esto en época pre-pandemia).

Según el Reporte de Calidad de Aire Mundial al año 2020, Santiago de Chile se encuentra en el lugar número 26 del ranking de las capitales (3). Al considerar solo Latinoamérica y el Caribe se suman distintas ciudades al sur de Chile con mayor contaminación aérea por el uso de leña como la principal fuente de calefacción y cocina. Por lo cual, la contaminación ambiental es un tema que requiere de revisión y de políticas públicas para poder entregar una mejora calidad de vida para la población tanto a nivel nacional como mundial.

\footnotetext{
Efectos deletéreos de contaminantes ambientales

Los efectos deletéreos producidos por la contaminación ambiental son múltiples y dependen del tipo de contaminante, concentración, tiempo de exposición y susceptibilidad de cada persona. Sin embargo, se han descrito
}

\section{RESUMEN}

La contaminación ambiental afecta a más del $90 \%$ de la población mundial, y es considerado un factor de morbi-mortalidad respiratoria desde mediados de 1900. Sin embargo, al día de hoy las políticas públicas no han logrado mejorar en forma sustancial la calidad del aire. Siendo causante de más de 3.7 millones de muertes anuales según datos OMS. Los niños son los principales afectados debido a diferencias anatómicas, fisiológicas y de estilos de vida. Dentro de las complicaciones más frecuentes se encuentran las infecciones respiratorias, pero además puede verse afectado el desarrollo neurológico, patologías oncológicas, enfermedades crónicas (diabetes y asma), alteraciones cardiovasculares, malformaciones congénitas y salud mental entre otros. En el siguiente artículo se revisará la evidencia científica en relación a la contaminación ambiental, como afecta al sistema inmune y el microbioma, generando un aumento en las infecciones respiratorias de los niños

Palabras claves: Contaminación ambiental, niños e infecciones respiratorias.

\section{ABSTRACT}

Environmental pollution affects more than $90 \%$ of the world population, and is considered a factor of respiratory morbidity and mortality since the mid-1900s. However, to date public policies have not substantially improved air quality. Being the cause of more than 3.7 million deaths annually according to data from the World Health Organization (WHO), children are more susceptible due to anatomical, physiological and lifestyle differences with adults. Respiratory infections are among the most frequent complications, but neurological development, oncological pathologies, chronic diseases (diabetes and asthma), cardiovascular disorders, congenital malformations and mental health, can be attributed to pollution. The following article will review the scientific evidence of environmental pollution, how it affects the immune system and the microbiome, generating an increase in respiratory infections in children.

Keywords: Environmental pollution, respiratory infections and children.

diversos mecanismos de daño que se resumen en la tabla 1.

\section{Niños y su mayor labilidad}

Los niños al estar en un periodo de periodo crecimiento y desarrollo intenso se ven afectados de forma más severa, además tienen mayor exposición ambiental por estar más tiempo al aire libre, tienen factores propios de fisiología y fisiopatología respiratoria e inmunológica que los hace más susceptibles (4). Todo esto se resumen en la Tabla 2.

\section{Contaminación extradomiciliaria}

Las principales fuentes de contaminación extradomiciliaria son las emisiones vehiculares, fábricas e industrias, plantas de energías y quema de biomasa. Estos producen diversos contaminantes, dentro de los cuales se encuentran:

- Material particulado (MP) que se clasifica según el tamaño de la partícula en MP10, MP 2.5 y MP 1.0 o ultrafinas, las cuales tendrán una absorción diversa en nuestro organismo, pudiendo las ultrafinas incluso traspasar la barrera hemato-encefálica.

- Ozono $\left(\mathrm{O}_{3}\right)$ : es un tipo de contaminante secundario, se produce mediante reacciones entre la luz ultravioleta en la estratósfera y los óxidos de nitrógeno y óxido de azufre.

- Monóxido de carbono (CO)

- Dióxido de azufre $\left(\mathrm{SO}_{2}\right)$

- Dióxido de nitrógeno $\left(\mathrm{NO}_{2}\right)$

- Plomo

De estos se establecen mediciones para evaluar la calidad del aire de MP2.5, MP 10, dióxido de $\mathrm{SO}_{2}, \mathrm{O}_{3}$ y NO 2 . En la tabla 3. se mencio-
Autor para correspondencia: Soledad Montes Franceschini Hospital Dra Eloisa Diaz - La Florida Froilán Lagos 6542, La Florida. RM. Chile Teléfono: +569-9539-5164
Correo electrónico solemf@yahoo.com 
nan los niveles de corte recomendados según las diversas organizaciones o países, incluido Chile.

Tabla 1. Efectos deletereos que produce al contaminación ambiental.

\begin{tabular}{|l|}
\hline Inducción estrés oxidativo $\left(\mathrm{NO}_{2}, \mathrm{O}_{3}, \mathrm{MP}\right)$ \\
\hline $\begin{array}{l}\text { Efectos pro-inflamatorios (metales pesados, } \\
\mathrm{MP} \text { y } \mathrm{NO}_{2} \text { ) }\end{array}$ \\
\hline $\begin{array}{l}\text { Efectos genotóxicos (MP, hidrocarburos } \\
\text { aromáticos y benzopirenos) }\end{array}$ \\
\hline Inducción de cambios epigenéticos \\
\hline $\begin{array}{l}\text { Alteración del sistema endocrinológico (MP, } \\
\text { hidrocarburos aromáticos y metales pesados) }\end{array}$ \\
\hline $\begin{array}{l}\text { Disminución de péptidos y proteínas antimi- } \\
\text { crobianas }\end{array}$ \\
\hline $\begin{array}{l}\text { Activación del eje hipotálamo-hipófisis- } \\
\text { adrenal }\end{array}$ \\
\hline Alteraciones del microbioma \\
\hline
\end{tabular}

$\mathrm{NO}_{2}$ : dioxido de nitrógeno, $\mathrm{O}_{3}$ : ozono, MP: material particulado

Tabla 2. Factores del niño que aumentan el riesgo de los contaminantes ambientales.

\begin{tabular}{|l|}
$\begin{array}{l}\text { Sistema inmune inmaduro, con posibilidad de } \\
\text { cambio de respuesta Th2 }\end{array}$ \\
\hline $\begin{array}{l}\text { Aumento de frecuencia respiratoria con mayor } \\
\text { ventilación y por ende mayor absorción de } \\
\text { partículas aéreas (inhalan más aire por unidad } \\
\text { de kilo) }\end{array}$ \\
\hline Mayor resistencia de la vía aérea periférica \\
\hline $\begin{array}{l}\text { Menor volumen pulmonar y menor superficie } \\
\text { alveolar }\end{array}$ \\
\hline Menor efectividad de la tos \\
\hline $\begin{array}{l}\text { Mayor cantidad de células secretoras, lo que } \\
\text { conlleva a hipersecreción frente a diversas } \\
\text { noxas }\end{array}$ \\
\hline
\end{tabular}

\section{Contaminación intradomiciliaria}

La contaminación intradomiciliaria, es la principal fuente de contaminación a la cual las personas de países occidentales están expuestas; ya que según estudios previo a la pandemia, alrededor del $80 \%$ de las actividades eran dentro de la casa, colegios u oficinas (5).

La calidad del aire intradomiciliario está influida por los contaminantes extra-domiciliarios, la calidad y cantidad de la ventilación, la presencia de alergenos intradomiciliarios y las actividades propias del hogar. Dentro de estas últimas se encuentran el tabaquismo, consumo de cigarrillo electrónicos, tipo de calefacción y cocina (gas, leña, carbón, parafina). Otros con-

Tabla 3. Niveles de corte que se consideran perjudiciales para la salud para los contaminantes ambientales.

\begin{tabular}{|c|c|c|c|c|}
\hline & OMS & UE & CHILE (2022) & EEUU \\
\hline MP2.5 & $\begin{array}{c}10 \mathrm{ug} / \mathrm{m}^{3} \text { promedio anual } \\
25 \mathrm{ug} / \mathrm{m}^{3} 24 \mathrm{~h} \max \end{array}$ & $\begin{array}{l}25 \mathrm{ug} / \mathrm{m}^{3} \\
\text { promedio anual }\end{array}$ & $\begin{array}{c}20 \mathrm{ug} / \mathrm{m}^{3} \\
\text { promedio anual } \\
50 \mathrm{ug} / \mathrm{m}^{3} 24 \mathrm{~h}\end{array}$ & $\begin{array}{c}35 \mathrm{ug} / \mathrm{m}^{3} \\
\text { promedio } 24 \mathrm{~h}\end{array}$ \\
\hline MP10 & $\begin{array}{c}20 \mathrm{ug} / \mathrm{m}^{3} \text { promedio anual } \\
50 \mathrm{ug} / \mathrm{m}^{3} \text { promedio día }\end{array}$ & $\begin{array}{c}40 \mathrm{ug} / \mathrm{m}^{3} \\
\text { promedio anual } \\
50 \mathrm{ug} / \mathrm{m}^{3} \\
\text { promedio día }\end{array}$ & $\begin{array}{c}50 \mathrm{ug} / \mathrm{m}^{3} \\
\text { promedio anual } \\
120 \mathrm{ug} / \mathrm{m} \\
\text { promedio } 24 \mathrm{~h}\end{array}$ & $\begin{array}{c}150 \mathrm{ug} / \mathrm{m}^{3} \\
\text { promedio } 24 \mathrm{~h}\end{array}$ \\
\hline $\mathrm{NO}_{2}$ & $\begin{array}{c}40 \mathrm{ug} / \mathrm{m}^{3} \mathrm{promedio} \text { anual } \\
200 \mathrm{ug} / \mathrm{m}^{3} 1 \mathrm{~h} \max \end{array}$ & $\begin{array}{c}40 \mathrm{ug} / \mathrm{m}^{3} \\
\text { promedio anual } \\
200 \mathrm{ug} / \mathrm{m} 31 \mathrm{~h} \text { max }\end{array}$ & $\begin{array}{c}100 \mathrm{ug} / \mathrm{m}^{3} \text { o } 53 \mathrm{ppb} \\
\text { promedio anual }\end{array}$ & $\begin{array}{c}53 \mathrm{ppb} \\
\text { promedio anual }\end{array}$ \\
\hline $\mathrm{O}_{3}$ & $100 \mathrm{ug} / \mathrm{m}^{3} 8 \mathrm{~h} \max$ & $120 \mathrm{ug} / \mathrm{m}^{3} 8 \mathrm{~h} \max$ & $120 \mathrm{ug} / \mathrm{m}^{3} 8 \mathrm{~h} \max$ & $\begin{array}{l}0.070 \mathrm{ppm} \\
\text { promedio } 8 \mathrm{~h}\end{array}$ \\
\hline $\mathrm{SO}_{2}$ & $\begin{array}{c}20 \mathrm{ug} / \mathrm{m}^{3} \text { al día } \\
500 \mathrm{ug} / \mathrm{m}^{3} 10 \mathrm{~min} \max \end{array}$ & $\begin{array}{l}125 \mathrm{ug} / \mathrm{m}^{3} \mathrm{prom} \text { día } \\
350 \mathrm{ug} / \mathrm{m}^{3} 1 \mathrm{~h} \max \end{array}$ & $\begin{array}{c}60 \mathrm{ug} / \mathrm{m}^{3} \text { o } 23 \mathrm{ppb} \\
\text { promedio anual }\end{array}$ & $0.5 \mathrm{ppm}$ prom $3 \mathrm{~h}$ \\
\hline
\end{tabular}

MP2.5: material particulado 2.5, MP10: material particulado 10, $\mathrm{NO}_{2}$ : dióxido de nitrógeno, $\mathrm{O}_{3}$ : ozono, $\mathrm{SO}_{2}$ : dióxido de azufre ug $/ \mathrm{m}^{3}$ : microgramo por metro cúbico, h: hora, max: máximo, ppb: partes por billón, OMS: Organización Mundial de la Salud UE: Unión Europea, EEUU: Estados Unidos.

taminantes son los componentes orgánicos volátiles provenientes de materiales de construcción, productos de limpieza, cosmética, desodorantes ambientales, etc (5). Los niños pueden pasar más de 8 horas al día en el colegio por lo cual se debe considerar este ambiente dentro las causas de contaminación al igual que el trayecto de ida y regreso al mismo. Existen estudios que demuestran que dentro de los colegios hay caspa de ratón, perro, gato, diversos hongos, $\mathrm{MP} 2.5$ y $\mathrm{O}_{3}(6)$.

\section{Contaminación ambiental y sistema inmune}

El ingreso del MP a la vía respiratoria produce alteraciones del mucus con pérdida de sus antioxidantes, disrupción de la integridad del epitelio con aumento de la permeabilidad cambios en los receptores de los macrófagos alveolares con alteración de su fagocitosis, aumento en la migración de eosinófilos, neutrófilos y mastocitos al epitelio, y modulación de citoquinas y quimiocinas todo lo cual resulta en un estado proinflamatorio y aumento del estrés oxidativo $(7,8)$

Dentro de los cambios del sistema inmunológico adaptativo, la exposición de MP, carbón y/o diésel, producen un cambio en el balance de respuesta inmune Th1 / Th2. A su vez, las citoquinas inflamatorias junto con el aumento de mucus e inflamación crónica producen cambios en los isotipos de inmunoglobulinas, aumentando la producción de inmunoglobulina E y disminución de inmunoglobulina A por los linfocitos B (7). Por otro lado, las células dendríticas expuestas a MP producen una menor cantidad de células efectoras Th1, por una maduración atenuada; siendo así el individuo más susceptible a diversos patógenos (7).
Esto genera una disregulación de la actividad antiviral y antimicrobiana por exposición a distintos contaminantes. A modo de ejemplo, los macrófagos saturados de plomo tienen menor actividad fagocítica, la exposición de dióxido de nitrógeno aumenta la la expresión de moléculas de adhesión ICAM-1, lo cual se suma al desbalance de la respuesta Th1 / Th2 favoreciendo a ésta última frente a diversos patógenos (7).

En resumen, el aire contaminado ingresa y produce alteraciones a nivel del sistema inmune innato y el adaptativo lo cual favorece una respuesta inflamatoria Th2 con una respuesta Th1 inhibida. La exposicón crónica de macrófagos favorece su maduración en macrófagos tipo 2, con un aumento de respuesta de hipersensibilidad retardada, inflamación pulmonar crónica y enfisema pulmonar (8).

\section{Contaminación ambiental y microbioma}

Durante los últimos años, el estudio del microbioma respiratorio y sus diferencias según distinta patologías crónicas respiratorias ha aumentado en forma exponencial. En un individuo sano predominan la Prevotella, Streptococcus, Veillonella, Fusobacterium, Porphyromonas, y Neisseria (9). Diversos estudios han demostrado que la exposición a MP2.5, MP10, $\mathrm{SO}_{2}, \mathrm{NO}$ y $\mathrm{O}_{3}$ en individuos sanos cambian la composición del microbioma observándose un aumento de Streptococcus, Staphylococcus, Haemophilus, Moraxella, Leptotrichia y Petrobacter asociado a una disminución de Prevotella, Veillonella, Fusobacterium, Camphylobacter, Capnocytophaga, Neisseria y Tropheryma (9), sin embargo a la fecha no se sabe las implicancias clínicas que esto podría tener y es material en estudio. 


\section{Contaminación ambiental e infecciones}

Como ya se mencionó anteriormente, el estrés oxidativo producido por la exposicón a contaminantes ambientales, altera el sistema inmune favoreciendo un aumento de las infecciones virales y bacterianas.

Un metanálisis reciente correlaciona la exposición de contaminantes provenientes de termoeléctricas a carbón con la salud infantil, demostrando que la exposición a $\mathrm{NO}_{2}, \mathrm{SO}_{2}, \mathrm{O}_{3}$, $\mathrm{CO}$ e hidrocarburos, se asocia a un aumento de síntomas respiratorios (tos, sibilancias y apremio respiratorio) con mayor número de consultas en urgencias y menor función pulmonar a largo plazo (10).

La exposición a MP2.5 y MP10 se asocia a un aumento de infecciones virales en menores de 7 meses y en preescolares respectivamente. Además de producir un aumento en las infecciones, se produce un aumento de la severidad, mayor riesgo de complicaciones y de días de hospitalización (exposición a mayores niveles de $\mathrm{O}_{3}$ y MP 10). Por otro lado, la mayor exposición a NO, aumenta la susceptibilidad, severidad y tiempo de recuperación de infecciones virales (11).

Un estudio realizado en China, demostró una asociación entre aumento de $10.7 \%$ de los cuadros gripales con niveles mayores de MP2.5, asociado a temperaturas más bajas (12). Otro estudio en Brisbane, Australia, relaciona el aumento de MP10 y temperaturas promedios más bajas con aumento de influenza en niños; niveles altos de $\mathrm{O}_{3}$ también se correlacionó con cuadros de influenza, independiente de la temperatura (13). Esto podría deberse a un aumento de la sobrevida viral, estabilidad del virus y transmisibilidad (11), asociado a una mayor susceptibilidad individual por las alteraciones inmunológicas ya mencionadas

En relación con el virus respiratorio sincicial (VRS), un estudio realizado en Italia, que abarcó 10 temporadas de VRS, asocia su aumento a menores temperaturas y mayor humedad relativa; sin embargo, al mantener estos factores clímaticos constantes, el aumento de benzeno se asocia a aumento de VRS (14). El benzeno produciría daño tóxico en el epitelio respiratorio que llevaría a cambios apoptóicos celulares. Otro estudio de Lombardía, Italia, que incluyó las altas hospitalarias en menores de 1 año de 2 temporadas de VRS, asoció el aumento MP10 con más hospitalizaciones por bronquiolitis en esos periodos (15).

El aumento de MP10 con temperaturas cálidas se asocia a síntomas de resfrío común en preescolares (11).

La exposición a $\mathrm{O}_{3}$ aumenta la proteolisis, lo que favorece la infeccion por metapneumovirus y coronavirus (SARS). Al ser un conta- minante de alto poder oxidante, favorece las infecciones bacterianas y fúngicas en las vías aéreas (11)

En relación con la pandemia actual por coronavirus SARS-COV2 se ha postulado que los contaminantes ambientales podrían favorecer la sobrevida viral, ser un transportador del virus y fomentar su virulencia. Sin embargo, esto no se ha podido demostrar y al momento, se ha observado que la cuarentena se asocia a una disminución de la contaminación y a la vez disminución en la mortalidad por SARS-COV2 (11), pero falta la evidencia para evaluar causalidad.

Finalmente, la tuberculosis es endémica en muchos países, y la contaminación ambiental favorece su transmisión y capacidad de producir infección. El aumento de MP disminuye la fagocitosis de los macrófagos y la producción de interferón gamma y TNF-alfa, con aumento de citoquinas antiinflamatorias, lo cual favorece el desarrollo de tuberculosis (8).

\section{CONCLUSIÓN}

La contaminación ambiental influye de diversas formas en la salud de las personas, siendo los niños, las mujeres embarazadas y los adultos mayores los más afectados. Pese a la cantidad de evidencia científica que demuestran el aumento de morbi-mortalidad, los cambios en las políticas públicas han sido lentos y de difícil implementación y cumplimiento, lo cual mantiene a nuestro país y muchos otros con mala calidad de aire y por ende a nuestros niños expuestos a riesgos que tienen implicancias a corto, mediano y largo plazo. Para empezar, es fundamental crear una cultura de cuidado del ambiente y disminuir la contaminación generada dentro de nuestro hogar para poder entregar a las generaciones futuras un mejor planeta.
REFERÉNCIAS

1. Laborde A, Tomasina F, Bianchi F, Brune MN, Buka Comba P, et tal. Children's Health in Latin America: The Influence of Environmental Exposures. Environ Health Persp 2015;123(3): 201-9. http://dx.doi.org/10.1289/ ehp.1408292.

2. Sun Z, Zhu D. Exposure to outdoor air pollution and its human health outcomes: A scoping review. PLoS ONE 2019;14(5):e0216550. https://doi.org/10.1371/journal. pone. 0216550

3. World Air Quality Report, 2020. [Internet]. Switzerland: IQAir; 2021. Available from: https://www.iqair.com/es/ world-air-quality-report.

4. Oyarzún M. Contaminación aérea y sus efectos en la salud. Rev Chil Enf Respir 2010;26:16-25.

5. Eguiluz-Gracia I, Mathioudakis AG, Bartel S, Vijverberg $\mathrm{SJH}$, Fuertes $\mathrm{E}$, Comberiati $\mathrm{P}$, et tal. The need for clean air: The way air pollution and climate change affect allergic rhinitis and asthma. Allergy 2020;75:2170-2184 Doi10.1111/all.14177.

6. Akar-Chibril N, Phipatanakul W. The indoor environment and childhood asthma. Curr Allergy Asthma Rep 2020;20:43-59. https://doi.org/10.1007/s11882-02000941-5.

7. Glencross DA, Ho TR, Camiña N, Hawrylowicz CM, Pfeffer $P$. Air pollution and its effects on the immune system Free Radic Biol Med 2020;151:56-68. https://doi.org/10.1016/j.freeradbiomed.2020.01.179

8. Nagappan A, Bum Park S, Lee SJ, Moon Y. Mechanistic implications of biomass-derived particulate matter for immunity and immune disorders. Toxics 2021;9:18-39. https://doi.org/10.3390/toxics9020018.

9. Xue Y, Chu J, Li Y, Kong X. The influence of air pollution on respiratory microbiome: a link to respiratory disease. Toxicology Letters 2020, https://doi.org/10.1016/j.toxlet.2020.09.007.

10. Cortés S, Yohannessen K, Tellerias L, Ahumada E. Exposición a contaminantes provenientes de termoeléctricas a carbón y salud infantil:¿Cuál es la evidencia internacional y nacional? Rev Chil Pediatr 2019;90(1):102-14. Doi:10.32641/rchped.v90i1.748

11. Domingo IL, Rovira J. Effects of air pollutants on the transmission and severity of respiratory viral infections. Environ Res 2020;187:109650. https://doi.org/10.1016/j. envres.2020.109650.

12. Feng C, Li J, Sun W, Zhang Y, Wang Q. Impact of ambient fine particulate matter (PM2.5) exposure on the risk of influenza-like-illness: a time-series analysis in Beijing, China. Environ Health 2016;15:17. https://doi:10.1186/ s12940-016-0115-2

13. Xu Z, Hu W, Williams G, Clements AC, Kan H, Tong S. Air pollution, temperature and pediatric influenza in Brisbane, Australia. Environ Int 2013;59:384-9. https:// doi:10.1016/j.envint.2013.06.022.

14. Nenna R, Evangelisti M, Frassanito A, Scagnolari C, Pierangeli $A$, Antonelli $A$, et tal. Respiratory syncytial virus bronchiolitis, weather conditions and air pollution in an Italian urban area: An observational study. Environ Res 2017;158:188-93. https://dx.doi.org/10.1016/j.envres.2017.06.014.

15. Carugno M, Dentali F, Mathieu G, Fontanella A, Mariani J, Bordini L, et tal. PM10 exposure is associated with increased hospitalizations for respiratory syncytial virus bronchiolitis among infants in Lombardy, Italy. Environ Res 2018;166:452-7. https://doi:10.1016/j.envres,2018.06.016 